\title{
Food Insecurity and Eating Disorders: a Review of Emerging Evidence
}

\author{
Vivienne M. Hazzard ${ }^{1}$ (D) Katie A. Loth $^{2} \cdot$ Laura Hooper $^{3} \cdot$ Carolyn Black Becker $^{4}$
}

Accepted: 19 October 2020 / Published online: 30 October 2020

(C) Springer Science+Business Media, LLC, part of Springer Nature 2020

\begin{abstract}
Purpose of Review This review summarizes emerging evidence for the relationship between food insecurity and eating disorder (ED) pathology, outlines priorities for future research in this area, and comments on considerations for clinical and public health practice.

Recent Findings Among adults, food insecurity is cross-sectionally associated with higher levels of overall ED pathology, binge eating, compensatory behaviors, binge-eating disorder, and bulimia nervosa. Evidence for similar relationships among adolescents has been less robust; however, compared to studies of adults, there have been substantially fewer studies conducted in adolescents to date.

Summary Emerging evidence consistently indicates that food insecurity is cross-sectionally associated with bulimic-spectrum ED pathology among adults. Findings emphasize the need for ED research to include marginalized populations who have historically been overlooked in the ED field. Much more research is needed to better understand the relationship between food insecurity and ED pathology and to determine effective ways to intervene.
\end{abstract}

Keywords Eating disorders $\cdot$ Disordered eating $\cdot$ Food insecurity $\cdot$ Health inequities $\cdot$ Marginalized populations $\cdot$ Social determinants of health

\section{Introduction}

Food insecurity is characterized by limited or uncertain means to access nutritious food in a safe and socially acceptable manner [1]. The food security status of a household exists on a continuum ranging from high food security (i.e., consistent access to adequate food) to very low food security (i.e., reduced food intake among one or more household members, with adults' intake typically affected before children's intake) $[2,3]$. Food insecurity is a global problem that affects not only

This article is part of the Topical Collection on Eating Disorders

Vivienne M. Hazzard

viviennehazzard@gmail.com

1 Sanford Center for Biobehavioral Research, 120 Eighth Street South, Fargo, ND 58103, USA

2 Department of Family Medicine and Community Health, University of Minnesota Medical School, Minneapolis, MN, USA

3 Division of Epidemiology and Community Health, University of Minnesota School of Public Health, Minneapolis, MN, USA

4 Department of Psychology, Trinity University, San Antonio, TX, USA low- and middle-income countries, but also high-income countries such as the United States (U.S.) [4]. For instance, after experiencing a substantial rise in levels of food insecurity with the 2007-2009 recession, the U.S. returned to prerecession levels a decade later - with 14.3 million U.S. households $(11.1 \%)$ determined to be food-insecure in 2018 [3] only to experience another surge in food insecurity levels with the COVID-19 pandemic [5]. Importantly, recent increases in food insecurity secondary to the COVID-19 pandemic are (a) not limited to the U.S. and (b) unlikely to resolve until the global economic impact of the pandemic is reversed, as increased poverty is a major driver of decreased food access [6]. In summary, the points addressed in this review will likely continue to be particularly relevant in the coming years.

Food insecurity is a social determinant of health that disproportionately affects racial/ethnic minorities and lowincome households [3]. A sizeable body of evidence indicates that food insecurity is associated with adverse physical health outcomes, including type 2 diabetes and hypertension [7, 8], as well as with adverse mental health outcomes, including depression and anxiety [9]. Researchers also have investigated the association between food insecurity and weight status [10-12]; this line of research has produced mixed results. Much of the research on food insecurity and weight status 
has focused on higher weight status, finding some evidence in support of such an association with more robust evidence among women than men or children [10, 11]. Some evidence, however, indicates that greater severity of food insecurityparticularly in low-income countries - is associated with underweight status [12].

Despite considerable research examining food insecurity in relation to other health- and weight-related outcomes, the relationship between food insecurity and eating disorder (ED) pathology has been largely unexplored until recently. EDs (e.g., anorexia nervosa, bulimia nervosa, binge-eating disorder) represent a significant public health concern, as they are associated with substantial psychosocial impairment, psychiatric comorbidity, medical complications, and suicidality [13-15]. Even in the absence of meeting full diagnostic criteria, disordered eating attitudes and behaviors are of concern, given that they are associated with poorer dietary intake $[16,17]$, greater depressive symptoms [18, 19], suicidality $[20,21]$, and increased risk for the development of fullthreshold EDs [22, 23]. The overall lifetime prevalence of full-threshold EDs in the U.S. is estimated to be 6.4\% [24], and EDs affect people of all genders, ages, races, ethnicities, socioeconomic backgrounds, and weight statuses [13, 15, 25, 26].

One factor that appears to have contributed to the slow investigation of food insecurity and ED pathology is the ED stereotype. Although we now have evidence to the contrary $[13,15,25,26]$, EDs were historically believed to primarily affect "skinny, white, affluent girls" [27]. This misconception is commonly referred to as the SWAG stereotype [27], and unfortunately, it has shaped approaches to ED assessment, prevention, and treatment, as well as etiologic models. For instance, ED assessment tools used extensively today were typically developed and validated in samples that largely fit the SWAG stereotype [28, 29]. In addition, and of particular relevance to our understanding of food insecurity and ED pathology, items assessing key constructs were sometimes developed in a limiting manner based on this stereotype. As an example, dietary restraint (i.e., intentional attempts to restrict food intake) is considered to be a key feature of EDs [30]. This construct is also of interest to the food insecurity field; food insecurity assessment tools include items pertaining to cutting the size of meals, skipping meals, and being hungry but not eating $[31,32]$. Notably, however, ED assessment tools are limited to only assessing dietary restraint motivated by weight/shape concerns [28, 29], which is the key reason individuals who fit the SWAG stereotype restrict food intake. In contrast, food-insecure individuals engage in dietary restraint for reasons other than weight/shape concerns (e.g., to ensure children in the household have enough food) more than they do for weight/shape reasons [33]. Therefore, although dietary restraint is an obvious parallel between food insecurity and ED pathology, this parallel has been overlooked in much of the research within the ED field secondary to a focus on ED presentations consistent with the SWAG stereotype.

An association between food insecurity and ED pathology broadly was first identified by Kendall and colleagues in 1996 [34], but this body of literature did not begin to truly grow until two decades later. This review aims to (a) provide context for why food insecurity might influence ED risk, (b) summarize emerging evidence for the relationship between food insecurity and ED pathology, (c) reflect on how this evidence fits with our understanding of EDs, (d) outline priorities for future research in this area, and (e) comment on considerations for clinical and public health practice. While this review focuses primarily on food insecurity and related policies in the U.S. because the relevant research to date has been conducted within the U.S., we hope to see research on this topic conducted in other areas of the world.

\section{Fluctuations in Food Availability: a Potential Eating Disorder Risk Factor}

Many individuals living with food insecurity experience a "feast-or-famine" cycle in which food intake oscillates according to fluctuations in food availability, such that food intake decreases during periods of food scarcity and increases during periods of relative food abundance (e.g., after receiving a paycheck) [11]. This cycle may be further amplified by some approaches used to mitigate food insecurity. As one example, the U.S. Supplemental Nutrition Assistance Program (SNAP) inadvertently exacerbates this cycle by providing benefits once per month. These benefits are often redeemed disproportionately soon after their receipt and exhausted before the end of the month $[11,35]$, resulting in food restriction.

Notably, attempts to restrict food, whether voluntary or involuntary, have been shown to result in a range of cognitive, emotional, and behavioral changes, including preoccupation with food, heightened emotional reactivity, and a tendency toward binge eating once restrictions are no longer in place [36]. A classic demonstration of these changes comes from the Minnesota Starvation Experiment led by Ancel Keys in 1944 1945 (i.e., prior to the establishment of the human subjects protections we have today). The purpose of Keys' study was to help inform nutritional rehabilitation efforts for individuals affected by famine during World War II; accordingly, the study consisted of baseline control, semi-starvation, and nutritional rehabilitation phases [37]. Volunteers who were deemed physically and mentally healthy at baseline developed intense preoccupation with food during the semi-starvation phase, and binge-eating behaviors emerged as more food was made available during the rehabilitation phase [37]. In line with these findings and the feast-or-famine cycle that many food-insecure individuals experience, qualitative research in low-income samples has highlighted patterns of food 
restriction and binge eating corresponding to fluctuations in food availability [38-40]. Food-insecure participants have described feeling immense excitement accompanying the influx of food after receiving a paycheck or SNAP benefits, particularly for favorite foods, as well as feeling out-of-control during overeating episodes [39], hence characterizing such episodes as binge-eating episodes [41]. Quantitative work in this area, however, has only recently begun to grow.

\section{Emerging Evidence for a Link Between Food Insecurity and Eating Disorder Pathology}

\section{Overall Eating Disorder Pathology}

In recent years, food insecurity has been found to be associated with higher levels of overall ED pathology in crosssectional studies of college students [42, 43], postpartum women of higher weight status [44], and adult clients of food pantries [45••, 46॰], with effect sizes ranging from small to medium across studies [47]. Specifically, when comparing participants with food insecurity versus food security, effect sizes were small, with a Cohen's $d$ of 0.31 [43], to medium, with Cohen's $d$ 's ranging from 0.55 [44] to 0.64 [42]. When comparing food pantry clients with severe food insecurity versus marginal food security, effect sizes were also medium, with Cohen's $d$ 's ranging from $0.58\left[46{ }^{\bullet}\right]$ to $0.76[45 \bullet \bullet]$.

\section{Disordered Eating Behaviors}

In addition to being identified as a correlate of overall ED pathology, food insecurity has been found to be associated with specific disordered eating behaviors.

Binge Eating Among adults, food insecurity has consistently been found to be cross-sectionally associated with greater likelihood of binge eating across studies of a populationbased sample of emerging adults [48] and their parents [49], bariatric surgery candidates [50], and clients of food pantries $[45 \bullet \bullet, 46 \bullet]$. Participants with food insecurity were more likely to report binge eating than those who were food-secure among emerging adults $(25.3 \%$ versus $18.4 \%)$ [48], their parents (11.3\% versus $6.4 \%)$ [49], and bariatric surgery candidates (18.4\% versus $5.0 \%)$ [50]. Similarly, food pantry clients with severe food insecurity were more likely to report binge eating than those with marginal food security in the initial study by Becker and colleagues (16.4\% versus $7.9 \%$ ) [45••], as well as in their replication study (26.6\% versus $11.9 \%$ ) [46 ${ }^{\circ}$. Additionally, adults with food insecurity have been found to experience greater binge eating severity than those with food security, with a medium effect size $\left(\eta^{2}=0.08\right)$, as well as to objectively overeat more calories in a controlled ad libitum food intake paradigm than those with food security [51].
Existing research among adolescents, however, has not indicated an association between food insecurity and binge eating cross-sectionally [52•*], and while food insecurity during adolescence was found to predict $40 \%$ greater risk of new-onset binge eating 5 years later in one study, the difference was not statistically significant [53]. Collectively, findings provide robust support for an association between food insecurity and binge eating among adults, but the limited existing literature among adolescents is less clear.

Compensatory Behaviors Cross-sectional associations between food insecurity and compensatory behaviors (i.e., behaviors to counteract the effects of eating or attempt to control weight) have been observed in both adolescent $[52 \cdot \bullet]$ and adult samples [45••, 46•]. Hooper and colleagues found that adolescents living in food-insecure households were more likely than those living in food-secure households to report laxative use $(1.8 \%$ versus $0.7 \%)$, diuretic use $(1.9 \%$ versus $0.5 \%)$, fasting $(15.7 \%$ versus $11.0 \%)$, eating very little food (35.7\% versus $30.2 \%$ ), and skipping meals $(32.6 \%$ versus $26.3 \%$ ) for the purpose of controlling weight [52••]. Further, Becker and colleagues found striking differences in compensatory behaviors in both their initial and replication studies $\left[45 \bullet \bullet, 46{ }^{\circ}\right.$. Adult food pantry clients with severe food insecurity were more likely than those with marginal food security to report vomiting $(20.4 \%$ versus $2.6 \%$ [ $45 \cdot \bullet] ; 17.2 \%$ versus 9.5\% [46•]), laxative/diuretic use (22.8\% versus $2.6 \%$ [45••]; $12.3 \%$ versus $8.3 \%[46 \cdot])$, fasting $(50.2 \%$ versus $13.2 \%$ [45••]; $55.1 \%$ versus $15.9 \%[46 \bullet]$ ), and intense exercise (34.7\% versus $15.4 \%$ [45••]; $35.3 \%$ versus $20.6 \%$ [46•]) for the purpose of controlling weight or counteracting the effects of eating. Therefore, although the observed associations between food insecurity and compensatory behaviors were somewhat surprising and counter to the initial hypotheses of Becker and colleagues [45••], the general consistency in these findings across existing studies suggests these observed associations may in fact reflect true associations. Further, these findings align with observations from Keys' Minnesota Starvation Experiment, as some volunteers in the experiment experienced binge-eating episodes followed by vomiting during the rehabilitation phase $[37,54]$. It is worth noting, however, that Hooper and colleagues observed no differences by food security status for vomiting to control weight among adolescents $[52 \bullet \bullet]$.

\section{Eating Disorder Diagnoses}

Food insecurity has also been found to be cross-sectionally associated with specific ED diagnoses (as assessed via selfreport questionnaire) in an adult sample recruited online [55••, $56 \bullet \cdot]$. Using participants with body mass indexes less than $25 \mathrm{~kg} / \mathrm{m}^{2}$ as the referent group, Rasmusson and colleagues found food insecurity to be associated with binge-eating 
disorder [55••], and Lydecker and colleagues found food insecurity to be associated with bulimia nervosa $[56 \bullet \bullet]$. For simplicity and ease of interpretability in this review, we have used the raw numbers reported in these studies $[55 \cdot \bullet, 56 \bullet \cdot]$ to calculate prevalence estimates in the full sample of 1329 participants, irrespective of weight status. Binge-eating disorder was more common in participants with food insecurity $(8.6 \%)$ than those who were food-secure $(5.2 \%), \chi^{2}(1, N=$ $1329)=5.89, p=0.02$, and bulimia nervosa was also more common in participants with food insecurity $(9.1 \%)$ than those who were food-secure $(4.2 \%), \chi^{2}(1, N=1329)=13.07$, $p<0.001[55 \bullet \cdot, 56 \bullet \cdot$. These associations with bulimicspectrum EDs cohere with the aforementioned associations observed for binge eating and compensatory behaviors.

Childhood food neglect, a distinct but related construct to food insecurity, refers to restricted access to food due to caregivers neglecting their responsibilities to provide adequate food for their children, even if they have the financial resources to do so. Cross-sectional data from a nationally representative sample of U.S. adults were recently used to examine retrospectively reported childhood food neglect in relation to ED diagnoses (as assessed via clinical interview). Findings indicated that participants with a history of childhood food neglect were more likely than those without a history of childhood food neglect to have binge-eating disorder ( $3.5 \%$ versus $0.8 \%$ ), bulimia nervosa ( $0.6 \%$ versus $0.3 \%)$, and anorexia nervosa (2.8\% versus $0.8 \%$ ) [57]. Although food neglect and food insecurity are distinct experiences, they are related, and these findings provide further support for a link between limited access to food and ED pathology.

\section{State of the Evidence}

The studies reviewed above represent the extent of the existing empirical literature on food insecurity and ED pathology that we identified since database inception via searches of MEDLINE and PsycINFO in July 2020. Overall, we identified a total of fourteen studies quantitatively examining the link between food insecurity and either overall ED pathology, disordered eating behaviors, or ED diagnoses [34, 42-44, $45 \bullet \bullet, 46 \bullet, 48-51,52 \bullet \bullet, 53,55 \bullet \bullet, 56 \bullet$ ], eleven of which were published since 2017 [42, 43, 45••, 46•, 48, 50, 51, 52・•, 53, $55 \cdot \bullet, 56 \bullet \cdot$. Although this body of research is still small, it has grown tremendously in recent years relative to the limited attention this topic previously garnered.

Taken together, the evidence reviewed above lends quite consistent support for cross-sectional associations between food insecurity and ED pathology, particularly for bulimicspectrum ED pathology among adults. The evidence among adolescents is less robust; however, compared to studies of adults, there have been substantially fewer studies conducted in adolescents to date. With regard to restrictive-spectrum ED pathology, only the study of childhood food neglect has thus far explicitly examined associations with anorexia nervosa, and they did not parse out differences by restricting type versus binge-eating/purging type [57]. Therefore, less is known regarding the relationship between food insecurity and restrictive-spectrum ED pathology.

\section{Methodological Considerations}

Several methodological characteristics of the existing evidence should be considered. One such consideration is the samples in which existing studies were conducted. The majority of these studies were conducted in relatively large samples of greater than 500 participants $[43,44,45 \bullet \bullet, 46 \bullet, 48,49$, $52 \bullet, 53,55 \bullet \cdot 56 \bullet]$, thereby reducing the likelihood that observed associations represent false positive results due to low statistical power [58]. However, with a few exceptions in which samples were drawn from the population-based Project EAT (Eating and Activity over Time) studies [48, $49,52 \cdot \bullet, 53]$, the majority of these studies also utilized nonprobability samples (typically a mix of convenience and purposive sampling), resulting in lower generalizability of their findings.

Another consideration is the variety of measures used to assess food insecurity and ED pathology across studies. To assess food insecurity, most studies used some form of the U.S. Household Food Security Scale (HFSS), which has demonstrated reliability and convergent validity $[59,60]$ but relies upon retrospective recall, the impact of which on validity has not been evaluated. Some studies used the 18 -item full version of the HFSS [44], the 10-item adult version [43, 50], or the six-item short form $[49,51,52 \bullet \bullet, 55 \bullet, 56 \bullet \bullet]$. Other studies used the HFSS-derived two-item Hunger Vital Sign [42], a different pair of HFSS items [48], a single HFSS item [53], or the Radimer/Cornell Food Insecurity Measure [34, 45••, 46.]. A wide variety of measures were used to assess ED pathology; these included a single item to assess binge eating [48], as well as the Eating Attitudes Test [44], Eating Disorder Examination-Questionnaire [42], and Eating Disorder Diagnostic Scale [45••, 46•] to assess overall ED pathology. The Questionnaire on Eating and Weight Patterns has also been used to assess specific ED diagnoses [55••, 56••]. Given these differences in measurement, along with important differences in sample characteristics across studies, prevalence estimates of specific behaviors and diagnoses cannot be meaningfully compared across studies. However, the overall consistency in findings across studies despite these methodological differences lends confidence to the results.

An additional key methodological consideration is that with three exceptions, the vast majority of these studies were cross-sectional in nature. Regarding the three exceptions to this pattern, one study examined retrospectively reported history of food insecurity prior to the past year [42], one study conducted prospective analyses without accounting for 
baseline ED pathology [44], and one truly longitudinal study with non-significant findings examined new-onset ED pathology [53]. Importantly, none of these studies offer clarity on the temporality of the relationship between food insecurity and ED pathology.

Regarding potential confounders of the relationship between food insecurity and ED pathology, most estimates reported above (e.g., effect sizes, prevalences) were unadjusted, but associations remained statistically significant in a number of these studies after adjusting for socioeconomic factors such as education level and income $[43,48-51,52 \bullet \bullet, 55 \bullet, 56 \bullet \cdot$. In other words, in several of these studies, food insecurity was found to be associated with ED pathology above and beyond the contribution of socioeconomic status broadly, suggesting that food insecurity is not simply a proxy for socioeconomic status but rather may play a unique role in relation to ED risk. Another important consideration is that of the role of weight status, given that weight status has demonstrated associations with both food insecurity [10-12] and ED pathology [61]. Only two of the reviewed studies adjusted for weight status as a covariate, and those that did had non-significant results $[44,53]$. Caution should be used in conditioning on weight status - in other words, restricting the sample based on weight status, stratifying by weight status, or adjusting for weight status - in studies examining the relationship between food insecurity and ED pathology because weight status may be a common effect, or "collider," of both food insecurity and ED pathology [62]. Thus, conditioning on weight status may introduce selection bias [62]. Alternatively, weight status may act as a mediator, in which case adjusting for weight status could result in overadjustment bias [63], or it may act as a confounder, in which case adjusting for it would be appropriate. As the role of weight status may vary according to the specific research question, there is likely not a one-size-fits-all approach, but the role of weight status should be considered carefully in both the study design and analysis phases. Importantly, another layer of caution is needed concerning weight status for self-report studies. It is unclear to what degree low-income individuals in the U.S. know their weight, given that the two primary sources of weight information (i.e., home scales and medical appointments) may be luxuries for individuals who cannot afford sufficient food. To our knowledge, there is limited - if any-empirical evidence indicating that self-reported weight information is accurate in low-income populations such as those living with food insecurity. Lastly, we encourage researchers to remember that ED pathology is problematic regardless of weight status and that preliminary evidence suggests that weight stig$\mathrm{ma}$ is a concern in this marginalized population [45••]. As such, researchers should consider ethical issues around triggering weight stigma in addition to the potential inaccuracy of self-report weight data when conducting research with this oppressed population.
How Does This Evidence Fit in With What We Know About Eating Disorders?

Along with emerging research in other marginalized populations indicating that EDs do in fact affect racial/ethnic minorities [64], sexual minorities [65], gender minorities [66, 67], and individuals of higher weight status $[25,68]$, the findings reviewed above provide further evidence against the historical ED stereotype. However, because many existing theoretical models used to explain the onset and maintenance of EDs were developed largely in accordance with the SWAG stereotype, these models may need to be reworked to be more relevant for individuals who do not fit that stereotype. The dual pathway model of bulimic symptoms [69], which has substantial empirical support [69-72] and has helped inform an efficacious and effective preventive intervention program [73], is one such example. The dual pathway model posits that pressure for thinness and pursuit of the thin ideal increase risk for body dissatisfaction, which in turn increases risk for dieting and negative affect, which thereby increase risk for the onset of bulimic-spectrum ED pathology [69]. Body dissatisfaction has been found to be associated with food insecurity in both children [74] and adults [75] and is therefore likely still relevant in populations living with food insecurity. However, expanding the dual pathway model to include limited food availability as a parallel risk factor to body dissatisfaction in predicting dietary restraint and negative affect might provide greater predictive value in low-income populations. There is some evidence to support such pathways, as dietary restraint is associated with ED pathology even when that restraint is not motivated by weight/shape concerns [33, 46॰], and qualitative work suggests that the experience of food insecurity may evoke particular facets of negative affect, such as shame [76]. Therefore, individuals living with food insecurity may experience a dual burden within the dual pathway model in the sense that body dissatisfaction and limited food availability may both engender dietary restraint and negative affect, and, in turn, bulimic symptoms.

\section{Future Directions for Research}

Given that this area of research is still in its nascency, there are a multitude of avenues to be explored in future research. For one, studies are needed to elucidate the temporality and underlying mechanisms of the relationship between food insecurity and ED pathology; longitudinal work will be crucial for these aims. Considering that longitudinal evidence from other domains suggests the relationship between food insecurity and emotional health broadly is bidirectional [77], a bidirectional relationship between food insecurity and ED pathology is likely. Ways in which ED pathology might influence food insecurity could include unemployment due to functional 
impairment [78] and financial burdens associated with binge eating (i.e., food costs) and purging (e.g., laxative costs) [79]. With regard to underlying mechanisms on the pathway from food insecurity to ED pathology, there are undoubtedly a number of important mechanisms to explore. One such possibility might be to investigate the roles of stigma and discrimination, as food insecurity is associated with higher levels of weight stigma [45•*] and experiences of racial/ethnic discrimination [80]. In addition to elucidating temporality and underlying mechanisms, more work is needed to better understand the nature of the relationship between food insecurity and ED pathology with regard to factors such as development and ED presentation. Specifically, more research is needed among youth to clarify whether the strength of the association differs according to developmental stage (e.g., adolescence versus adulthood). Research is also needed to better understand the relationship between food insecurity and restrictive-spectrum ED pathology. In tandem with longitudinal research, qualitative research will be invaluable for learning more about EDs in the context of food insecurity because we cannot presume to understand the underlying processes without listening to the voices of individuals living these experiences. For example, qualitative research could help clarify why some individuals living with food insecurity engage in restrictive and compensatory behaviors, as these associations are theoretically less intuitive than the association between food insecurity and binge eating. It is important for researchers to recognize that thus far, much of the way we think about EDs has been informed by the ubiquitous SWAG stereotype; thus, it is critical that we listen to individuals living with food insecurity tell us about their experiences before we develop theoretical models to explain those experiences.

Beyond research to better understand the relationship between food insecurity and ED pathology, a critical area for future work lies in the intervention realm. A communitybased participatory research approach (i.e., working with community members as collaborators in the research process) will be instrumental in guiding this work. Multilevel interventions in particular hold promise in this area, as a multipronged approach will be necessary to intervene on food insecurity itself, and other effects of poverty, in addition to ED pathology. Relatedly, we need to invest in research to explore both the positive impacts and unintended consequences of existing and proposed approaches designed to reduce the impact of food insecurity. Current approaches tend to fall into one of two categories: public assistance policies (e.g., SNAP, the Special Supplemental Nutrition Program for Women, Infants, and Children [WIC], school meals) or communitylevel efforts (e.g., food pantries, community gardens) [81]. For approaches in both categories, we need to identify ways to maximize intervention benefits while minimizing any negative impacts (such as exacerbation of the feast-or-famine cycle with SNAP). Lastly, we will need to explore the extent to which interventions designed to address higher weight status in individuals living with food insecurity perpetuate weight stigma and increase risk for ED pathology.

\section{Clinical and Public Health Implications}

The evidence reviewed here lends itself to important clinical implications. First, healthcare providers should challenge assumptions about the SWAG stereotype so as not to overlook diagnoses in patients who fall outside of the stereotype. Along those lines, existing ED assessment tools should be reevaluated, considering the possibility of broadening dietary restraint assessment to include motivations other than weight/ shape concerns. Additionally, screening for food insecurity should be a routine component of the intake process for ED treatment, and the food security status of patients should be considered throughout the treatment plan. For example, meal planning - an important part of eating disorder treatmenthinges on patients being able to afford food. Importantly, secondary to shame, patients may not self-disclose their inability to afford their meal plan if they are not asked. Similarly, taking prescribed medications - also an important part of treatment for some patients - may not be feasible if patients have to make choices between paying for food or medications.

Regarding public health implications, the observed associations between food insecurity and ED pathology highlight a particular need for low-cost ED treatment and prevention efforts in food-insecure populations, as these populations have less resources to access expensive team-based treatment. We also need to target food insecurity itself, using evidenceinformed policies and community-level interventions. Further, as food insecurity is rooted in systemic inequities and structural barriers to basic human rights, we need to move further upstream and address underlying structural racism in order to eliminate racial/ethnic disparities in food insecurity [82].

\section{Conclusions}

In conclusion, recent evidence consistently supports crosssectional associations between food insecurity and ED pathology, particularly for bulimic-spectrum ED pathology among adults. This provides evidence against the historical ED stereotype and emphasizes the need to include marginalized populations in ED research. Much more research is needed in this area to better understand the relationship between food insecurity and ED pathology and determine effective ways to intervene at multiple levels.

Acknowledgments Vivienne Hazzard's time for this research was supported by the National Institute of Mental Health (grant number: 
T32MH082761, PI: Scott Crow). Laura Hooper's time was supported, in part, by the Health Resources and Services Administration (HRSA) of the U.S. Department of Health and Human Services (grant number: T71MC00006-40-00, PI: Renee Sieving). Katie Loth's time was supported, in part, by the National Institute of Child Health and Human Development (grant number: K23HD090324-01A1, PI: Katie Loth).

\section{Compliance with Ethical Standards}

Conflict of Interest The authors declare that they have no conflict of interest.

Human and Animal Rights All reported studies/experiments with human or animal subjects performed by the authors have been previously published and complied with all applicable ethical standards (including the Helsinki declaration and its amendments, institutional/national research committee standards, and international/national/institutional guidelines).

\section{References}

Papers of particular interest, published recently, have been highlighted as:

- Of importance

-• Of major importance

1. Anderson SA, editor. Core indicators of nutritional state for difficult-to-sample populations. J Nutr Life Sciences Research Office. 1990:1555-600.

2. United States Department of Agriculture Economic Research Service. Definitions of Food Security [Internet]. 2019. Available from: https://www.ers.usda.gov/topics/food-nutrition-assistance/ food-security-in-the-us/definitions-of-food-security/. Accessed 22 Jul 2020.

3. Coleman-Jensen A, Rabbitt MP, Gregory C, Singh A. Household food security in the United States in 2018. Washington, DC: ERR270, U.S. Department of Agriculture, Economic Research Service; 2019.

4. Jones AD. Food insecurity and mental health status: a global analysis of 149 countries. Am J Prev Med. 2017;53:264-73.

5. Waxman E, Gupta P, Karpman M. More than one in six adults were food insecure two months into the COVID-19 recession. 2020.

6. Laborde D, Martin W, Swinnen J, Vos R. COVID-19 risks to global food security. Science. 2020;369(80):500-2.

7. Weaver LJ, Fasel CB. A systematic review of the literature on the relationships between chronic diseases and food insecurity. Food Nutr Sci. 2018;9:519-41.

8. Abdurahman AA, Chaka EE, Nedjat S, Dorosty AR, Majdzadeh R. The association of household food insecurity with the risk of type 2 diabetes mellitus in adults: a systematic review and meta-analysis. Eur J Nutr. 2019;58:1341-50.

9. Arenas DJ, Thomas A, Wang JC, DeLisser HM. A systematic review and meta-analysis of depression, anxiety, and sleep disorders in US adults with food insecurity. J Gen Intern Med. 2019;34: 2874-82.

10. Larson NI, Story MT. Food insecurity and weight status among U.S. children and families: a review of the literature. Am J Prev Med. 2011;40:166-73.

11. Dinour LM, Bergen D, Yeh MC. The food insecurity-obesity paradox: a review of the literature and the role food stamps may play. J Am Diet Assoc. 2007;107:1952-61.
12. Moradi S, Mirzababaei A, Dadfarma A, Rezaei S, Mohammadi H, Jannat B, et al. Food insecurity and adult weight abnormality risk: a systematic review and meta-analysis. Eur J Nutr. 2019;58:45-61.

13. Hudson JI, Hiripi E, Pope HG, Kessler RC. The prevalence and correlates of eating disorders in the National Comorbidity Survey Replication. Biol Psychiatry. 2007;61:348-58.

14. Mitchell JE, Crow S. Medical complications of anorexia nervosa and bulimia nervosa. Curr Opin Psychiatry. 2006;19:438-43.

15. Swanson SA, Crow SJ, Le Grange D, Swendsen J, Merikangas KR. Prevalence and correlates of eating disorders in adolescents. Results from the national comorbidity survey replication adolescent supplement. Arch Gen Psychiatry. 2011;68:714-23.

16. Larson NI, Neumark-Sztainer D, Story M. Weight control behaviors and dietary intake among adolescents and young adults: longitudinal findings from Project EAT. J Am Diet Assoc. 2009;109: 1869-77.

17. Neumark-Sztainer D, Hannan PJ, Story M, Perry CL. Weightcontrol behaviors among adolescent girls and boys: implications for dietary intake. J Am Diet Assoc. 2004;104:913-20.

18. Stice E, Bearman SK. Body-image and eating disturbances prospectively predict increases in depressive symptoms in adolescent girls: a growth curve analysis. Dev Psychol. 2001;37:597-607.

19. Hazzard VM, Hahn SL, Bauer KW, Sonneville KR. Binge eatingrelated concerns and depressive symptoms in young adulthood: seven-year longitudinal associations and differences by race/ethnicity. Eat Behav. 2019;32:90-4.

20. Kim DS, Kim HS. Body-image dissatisfaction as a predictor of suicidal ideation among Korean boys and girls in different stages of adolescence: a two-year longitudinal study. J Adolesc Health. 2009;45:47-54.

21. Crow S, Eisenberg ME, Story M, Neumark-Sztainer D. Are body dissatisfaction, eating disturbance, and body mass index predictors of suicidal behavior in adolescents? A longitudinal study. J Consult Clin Psychol. 2008;76:887-92.

22. Stice E, Marti CN, Durant S. Risk factors for onset of eating disorders: evidence of multiple risk pathways from an 8-year prospective study. Behav Res Ther Elsevier Ltd. 2011;49:622-7.

23. Stice E, Gau JM, Rohde P, Shaw H. Risk factors that predict future onset of each DSM-5 eating disorder: predictive specificity in highrisk adolescent females. J Abnorm Psychol. 2017;126:38-51.

24. Deloitte Access Economics. Social and economic cost of eating disorders in the United States of America: Report for the Strategic Training Initiative for the Prevention of Eating Disorders and the Academy for Eating Disorders. 2020.

25. Lipson SK, Sonneville KR. Eating disorder symptoms among undergraduate and graduate students at 12 U.S. colleges and universities. Eat Behav. 2017;24:81-8.

26. Pike KM, Dunne PE, Addai E. Expanding the boundaries: reconfiguring the demographics of the "typical" eating disordered patient. Curr Psychiatry Rep. 2013;15:411.

27. Sonneville KR, Lipson SK. Disparities in eating disorder diagnosis and treatment according to weight status, race/ethnicity, socioeconomic background, and sex among college students. Int J Eat Disord. 2018;51:518-26.

28. Fairburn C, Beglin S. Assessment of eating disorders: interview or self-report questionnaire? Int J Eat Disord. 1994;16:363-70.

29. Cooper Z, Cooper PJ, Fairburn CG. The validity of the eating disorder examination and its subscales. Br J Psychiatry. 1989;154: $807-12$.

30. Fairburn CG, Cooper Z, Shafran R. Cognitive behaviour therapy for eating disorders: a "transdiagnostic" theory and treatment. Behav Res Ther. 2003;41:509-28.

31. Carlson SJ, Andrews MS, Bickel GW. Measuring food insecurity and hunger in the United States: development of a national benchmark measure and prevalence estimates. J Nutr. 1999;129:510S$6 \mathrm{~S}$. 
32. Kendall A, Olson CM, Frongillo EA. Validation of the Radimer/ Cornell measures of hunger and food insecurity. J Nutr. 1995;125: 2793-801.

33. Middlemass KM, Cruz J, Gamboa A, Johnson C, Taylor B, Gomez $\mathrm{F}$, et al. Food insecurity \& dietary restraint in a diverse urban population. Eat Disord. 2020:1-14. https://doi.org/10.1017/ S1368980020000464.

34. Kendall A, Olson CM, Frongillo EA. Relationship of hunger and food insecurity to food availability and consumption. J Am Diet Assoc. 1996;96:1019-24.

35. Wilde PE, Ranney CK. The monthly food stamp cycle: shopping frequency and food intake decisions in an endogenous switching regression framework. Am J Agric Econ. 2000;82:200-13.

36. Polivy J. Psychological consequences of food restriction. J Am Diet Assoc. 1996;96:589-92.

37. Keys A, Brožek J, Henschel A, Mickelsen O, Taylor HL. The biology of human starvation. (2 vols). University of Minnesota Press; 1950.

38. Bove CF, Olson CM. Obesity in low-income rural women: qualitative insights about physical activity and eating patterns. Women Heal. 2006;44:57-78.

39. Olson CM, Bove CF, Miller EO. Growing up poor: long-term implications for eating patterns and body weight. Appetite. 2007;49: 198-207.

40. Tester JM, Lang TC, Laraia BA. Disordered eating behaviours and food insecurity: a qualitative study about children with obesity in low-income households. Obes Res Clin Pract. 2016;10:544-52.

41. American Psychiatric Association. Feeding and eating disorders. Diagnostic Stat Man Ment Disord. Washington, DC: American Psychiatric Association Publishing; 2013.

42. Darling KE, Fahrenkamp AJ, Wilson SM, D'Auria AL, Sato AF. Physical and mental health outcomes associated with prior food insecurity among young adults. J Health Psychol. 2017;22:572-81.

43. El Zein A, Shelnutt KP, Colby S, Vilaro MJ, Zhou W, Greene G, et al. Prevalence and correlates of food insecurity among U.S. college students: a multi-institutional study. BMC Public Health. 2019;19:660.

44. Laraia B, Vinikoor-Imler LC, Siega-Riz AM. Food insecurity during pregnancy leads to stress, disordered eating, and greater postpartum weight among overweight women. Obesity. 2015;23:130311.

45.• Becker CB, Middlemass K, Taylor B, Johnson C, Gomez F. Food insecurity and eating disorder pathology. Int J Eat Disord. 2017;50: 1031-40 This was the first study to assess the full spectrum of ED pathology in a low-income population with food insecurity.

46. Becker CB, Middlemass KM, Gomez F, Martinez-Abrego A. Eating disorder pathology among individuals living with food insecurity: a replication study. Clin Psychol Sci. 2019;7:1144-58 This study replicated the findings observed by Becker and colleagues in 2017, including associations between food insecurity and compensatory behaviors.

47. Cohen J. Statistical power analysis for the behavioral sciences. 2nd ed. Hillsdale: Lawrence Earlbaum Associates; 1988.

48. Larson N, Laska MN, Neumark-Sztainer D. Food insecurity, diet quality, home food availability, and health risk behaviors among emerging adults: findings from the EAT 2010-2018 study. Am J Public Health. 2020:e1-7. https://doi.org/10.1017/ S1368980020000464.

49. Bruening M, MacLehose R, Loth K, Story M, Neumark-Sztainer D. Feeding a family in a recession: food insecurity among Minnesota parents. Am J Public Health. 2012;102:520-6.

50. Zickgraf HF, Stefano E, Price J, Veldheer S, Rogers A, Rigby A. The relationship between food insecurity and binge and night eating symptoms in prebariatric surgery patients is mediated by depressive symptoms. Surg Obes Relat Dis. 2019;15:1374-9.
51. Stinson EJ, Votruba SB, Venti C, Perez M, Krakoff J, Gluck ME. Food insecurity is associated with maladaptive eating behaviors and objectively measured overeating. Obesity. 2018;26:1841-8.

52.• Hooper L, Telke S, Larson N, Mason SM, Neumark-Sztainer D. Household food insecurity: associations with disordered eating behaviours and overweight in a population-based sample of adolescents. Public Health Nutr. 2020:1-10. https://doi.org/10.1017/ S1368980020000464. This study examined associations between household food insecurity reported by parents/ guardians and adolescent disordered eating behaviors in a racially/ethnically diverse population-based sample.

53. West CE, Goldschmidt AB, Mason SM, Neumark-Sztainer D. Differences in risk factors for binge eating by socioeconomic status in a community-based sample of adolescents: findings from Project EAT. Int J Eat Disord. 2019;52:659-68.

54. Eckert E, Gottesman I, Swigart S, Casper R. A 57-year follow-up investigation and review of the Minnesota Study on Human Starvation and its relevance to eating disorders. Arch Psychol. 2018;2:1-19.

55.• Rasmusson G, Lydecker JA, Coffino JA, White MA, Grilo CM. Household food insecurity is associated with binge-eating disorder and obesity. Int J Eat Disord. 2019;52:28-35 This was the first study to examine the association between food insecurity and binge-eating disorder.

$56 . \bullet$ Lydecker JA, Grilo CM. Food insecurity and bulimia nervosa in the United States. Int J Eat Disord. 2019;52:735-9 This was the first study to examine the association between food insecurity and bulimia nervosa.

57. Coffino JA, Grilo CM, Udo T. Childhood food neglect and adverse experiences associated with DSM-5 eating disorders in U.S. national sample. J Psychiatr Res. 2020;127:75-9.

58. Christley RM. Power and error: increased risk of false positive results in underpowered studies. Open Epidemiol J. 2010;3:16-9.

59. Keenan DP, Olson C, Hersey JC, Farmer SM. Measures of food insecurity/security. J Nutr Educ Behav. 2001;33:49-58.

60. Marques ES, Reichenheim ME, De Moraes CL, Antunes MML, Salles-Costa R. Household food insecurity: a systematic review of the measuring instruments used in epidemiological studies. Public Health Nutr. 2015;18:877-92.

61. Goldschmidt AB, Aspen VP, Sinton MM, Tanofsky-Kraff M, Wilfley DE. Disordered eating attitudes and behaviors in overweight youth, vol. 16: Obesity Blackwell Publishing Ltd; 2008. p. 257-64.

62. Cole SR, Platt RW, Schisterman EF, Chu H, Westreich D, Richardson D, et al. Illustrating bias due to conditioning on a collider. Int J Epidemiol. 2010;39:417-20.

63. Schisterman EF, Cole SR, Platt RW. Overadjustment bias and unnecessary adjustment in epidemiologic studies. Epidemiology. 2009;20:488-95.

64. Rodgers RF, Berry R, Franko DL. Eating disorders in ethnic minorities: an update. Curr Psychiatry Rep. 2018;20:90.

65. Calzo JP, Blashill AJ, Brown TA, Argenal RL. Eating disorders and disordered weight and shape control behaviors in sexual minority populations. Curr Psychiatry Rep. 2017;19:49.

66. Simone M, Askew A, Lust K, Eisenberg ME, Pisetsky EM. Disparities in self-reported eating disorders and academic impairment in sexual and gender minority college students relative to their heterosexual and cisgender peers. Int J Eat Disord. 2020;53:51324.

67. Lipson SK, Raifman J, Abelson S, Reisner SL. Gender minority mental health in the U.S.: results of a national survey on college campuses. Am J Prev Med. Elsevier. 2019;57:293-301.

68. Nagata JM, Garber AK, Tabler JL, Murray SB, Bibbins-Domingo $\mathrm{K}$. Prevalence and correlates of disordered eating behaviors among young adults with overweight or obesity. J Gen Intern Med. 2018;33:1337-43. 
69. Stice E. A prospective test of the dual-pathway model of bulimic pathology: mediating effects of dieting and negative affect. J Abnorm Psychol. 2001;110:124-35.

70. Stice E. Review of the evidence for a sociocultural model of bulimia nervosa and an exploration of the mechanisms of action. Clin Psychol Rev. 1994;14:633-61.

71. Stice E, Shaw HE. Role of body dissatisfaction in the onset and maintenance of eating pathology: a synthesis of research findings. J Psychosom Res. 2002;53:985-93.

72. Stice E, Van Ryzin MJ. A prospective test of the temporal sequencing of risk factor emergence in the dual pathway model of eating disorders. J Abnorm Psychol. 2019;128:119-28.

73. Stice E, Becker CB, Yokum S. Eating disorder prevention: current evidence-base and future directions. Int J Eat Disord. 2013;46:478 85.

74. Altman EA, Ritchie LD, Frongillo EA, Madsen KA. Food insecurity is associated with body dissatisfaction among children in California. J Acad Nutr Diet. 2019;119:1732-7.

75. Vedovato GM, Surkan PJ, Jones-Smith J, Steeves EA, Han E, Trude AC, et al. Food insecurity, overweight and obesity among low-income African-American families in Baltimore City: associations with food-related perceptions. Public Health Nutr. 2016;19: 1405-16.

76. Swales S, May C, Nuxoll M, Tucker C. Neoliberalism, guilt, shame and stigma: a Lacanian discourse analysis of food insecurity. $\mathrm{J}$
Community Appl Soc Psychol. 2020; casp.2475. https://doi.org/ 10.1017/S1368980020000464.

77. Bruening M, Dinour LM, Chavez JBR. Food insecurity and emotional health in the USA: a systematic narrative review of longitudinal research. Public Health Nutr. 2017;20:3200-8.

78. Samnaliev M, Noh HL, Sonneville KR, Austin SB. The economic burden of eating disorders and related mental health comorbidities: an exploratory analysis using the U.S. Medical Expenditures Panel Survey. Prev Med Reports. 2015;2:32-4.

79. Crow SJ, Frisch MJ, Peterson CB, Croll J, Raatz SK, Nyman JA. Monetary costs associated with bulimia. Int J Eat Disord. 2009;42: 81-3.

80. Phojanakong P, Brown Weida E, Grimaldi G, Lê-Scherban F, Chilton M. Experiences of racial and ethnic discrimination are associated with food insecurity and poor health. Int J Environ Res Public Health. 2019;16:4369.

81. Loopstra R. Interventions to address household food insecurity in high-income countries. Proc Nutr Soc. 2018;77:270-81.

82. Odoms-Young A, Bruce MA. Examining the impact of structural racism on food insecurity: implications for addressing racial/ethnic disparities. Fam Community Health. 2018;41(Suppl 2):139-48.

Publisher's Note Springer Nature remains neutral with regard to jurisdictional claims in published maps and institutional affiliations. 